Assistenzsysteme sowie die computergestützte Diagnostik und Therapie einen mittleren bis großen Einfluss haben werden. Dabei gehen sie davon aus, dass die Arzt-Patienten-Beziehung von den Veränderungen eher unberührt bleibt. Nur $18 \%$ der Fachärzte rechnen damit, dass die Vernetzung großen Einfluss auf ihre Beziehung zu den Patienten hat. Anders sieht das aus, wenn es um Gesundheitsplattformen und Portale geht: Sie werden nach Einschätzung von zwei Dritteln der Heilberufler das Arzt-PatientenVerhältnis in Zukunft stark prägen.

„Das Bild ist relativ homogen bei den verschiedenen Heilberufen“, berichtet Projektleiter Daniel Zehnich, stellvertretender Bereichsleiter Gesundheitsmärkte und -politik bei der apoBank. Es zeigen sich aber Schwerpunkte. So richten die Ärzte ein besonderes Augenmerk auf die Entwicklung bei den Assistenzsystemen. „Hier erwarten sie vergleichsweise starke Auswirkungen im Hinblick auf ihren beruflichen Alltag, die Arzt-Patienten-Beziehung und die Strukturen der
Gesundheitsversorgung sowie einen hohen Investitionsbedarf“", sagte Zehnich. Nur $9 \%$ rechnen allerdings mit einem hohen wirtschaftlichen Gewinn durch die Investitionen in Assistenzsysteme.

Die befürchtete Diskrepanz zwischen Kosten und Ertrag der Digitalisierung eint alle Heilberufler. Die Umfrage zeigt, dass Anreize für ein größeres Engagement bei der Digitalisierung fehlen. Haus- und Fachärzte wissen, dass die Digitalisierung keine Zukunftsmusik ist. Ein Drittel von ihnen geht davon aus, dass sie sich innerhalb der nächsten vier Jahre durchsetzen wird. Bei der Einschätzung der Folgen marschieren Standesorganisationen und ihre Mitglieder offensichtlich nicht im Gleichschritt. „Die Organisationen schätzen die Veränderungswucht geringer ein als die Heilberufler", sagt Zehnich. Insgesamt wird deutlich, dass die Heilberufler dem Thema Digitalisierung gegenüber eine hohe Sensibilität, aber keine genaue Vorstellung von den Auswirkungen im Detail haben.

Ilse Schlingensiepen

\title{
Operation ohne Einwilligung mit folgenschwerem Ausgang
}

\author{
Wo liegt nach einer Operation ohne wirksame Einwilligung von Patienten- \\ seite im Streit um mögliche negative Folgen die Beweislast? Der Bundes- \\ gerichtshof in Karlsruhe hat das in einem aktuellen Urteil klargestellt.
}

$\mathrm{m}$ entschiedenen Fall ging es um ein 2001 geborenes Mädchen, bei dem ein gutartiger Tumor festgestellt und das noch im Geburtsjahr operiert wurde. Der Tumor konnte aber nicht komplett entfernt werden. Später wurden zunehmende zystische Tumoranteile entdeckt. Spezialisten rieten davon ab, den Tumor möglichst weitgehend $\mathrm{zu}$ entfernen. Stattdessen empfahlen sie eine Drainierung. Die Eltern willigten in diese Operation ein. Der Operateur setzte sich jedoch darüber hinweg und versuchte eine vollständige Entfernung. Durch die Operation erlitt das Mädchen schwere Verletzungen und verstarb 2013.

Noch vor dem Tod reichten die Eltern für ihre Tochter eine Klage ein und forderten Schadensersatz sowie Schmerzensgeld in Höhe von 200.000 €. Das
Oberlandesgericht (OLG) Koblenz sprach ihnen aber nur $50.000 €$ Schadensersatz zu. Die Operation zur Entfernung des Hirntumors sei mangels Einwilligung zwar rechtswidrig gewesen und habe auch zu schweren Schäden geführt, nach Einschätzung einer Gutachterin sei es jedoch völlig unklar, wie sich das Mädchen ohne diese Operation entwickelt hätte. Weil insoweit die Beweispflicht bei den klagenden Eltern liege, sei hier vom ungünstigsten möglichen Verlauf auszugehen. Dieses Urteil hob der Bundesgerichtshof auf: Nach einer rechtswidrig ausgeführten Operation sei es "Sache des beklagten Arztes zu beweisen, dass der Patient ohne den rechtswidrig ausgeführten Eingriff dieselben Beschwerden haben würde", so die Richter (Az.: VI ZR 467/14). Martin Wortmann

\section{Alter darf nicht alleiniges Kriterium für die Zulassung sein}

Das Alter eines Mediziners darf nicht das alleinige Kriterium im Verfahren um die Besetzung eines Arztsitzes sein. Das hat selbst dann Gültigkeit, wenn einer der Bewerber bereits das Alter von 74 Jahren erreicht hat. So urteilte kürzlich das Sozialgericht Mainz (Az.: S 16 KA 211/14). Die Richter verwarfen mit ihrem Urteil die Auswahl eines 64-jährigen Augenarztes, der noch deutlich länger vertragsärztlich tätig hätte sein können. Das Gericht hat der Klage des 74-jährigen Konkurrenten, der unter Versorgungsgesichtspunkten besser geeignet war, stattgegeben und den Berufungsausschuss verpflichtet, erneut über die Zulassung zu entscheiden.

Martin Wortmann

\section{GOÄ wichtiger Punkt auf PKV-Reformagenda}

Die Novelle der GOÄ bleibt für die privaten Krankenversicherer (PKV) ein wichtiger Punkt auf der Reformagenda. Das hat der Vorsitzende des PKV-Verbands Uwe Laue auf der Jahrestagung im Juni in Berlin bestätigt. „Beide Seiten verhandeln weiter", berichtete er. „Wir sind aber Realisten genug, um zu erkennen, dass in dieser Legislaturperiode eine GOÄ-Novelle nicht mehr umgesetzt werden kann." Laue betonte zudem, dass das gemeinsam mit der Bundesärztekammer entwickelte GOÄ-Konzept eine deutliche Stärkung der sprechenden Medizin bringe.

Ilse Schlingensiepen

\section{IGeL: Verbraucherschützer rufen nach Schlichtungsstelle}

Die Verbraucherzentrale NordrheinWestfalen will den Umgang mit Selbstzahlerleistungen im Praxisalltag stärker reglementieren und fordert die Einrichtung einer unabhängigen, für Patienten leicht erreichbaren Schlichtungsstelle, die sich um Meinungsverschiedenheiten bei individuellen Gesundheitsleistungen (IGeL) kümmert. Die Aufsicht über diese Schiedsstelle obläge hierbei dem jeweils zuständigen Landesgesundheitsministerium. Details zur Ausgestaltung müssten per Verfahrensordnung für jedes Land geregelt werden, heißt es.

Matthias Wallenfels 